\title{
A Lorentzian-Function Approximation developed in calculating the charged-particle-induced nonresonant reaction rate
}

\author{
J.J. He ${ }^{\mathrm{a}}$ \\ School of Physics, University of Edinburgh, Mayfield Road, Edinburgh, EH9 3JZ, UK
}

Received: 29 June 2007

Published online: 30 July 2007 - (C) Società Italiana di Fisica / Springer-Verlag 2007

Communicated by G. Orlandini

\begin{abstract}
A Lorentzian-Function Approximation (LFA) has been developed in calculating the nonresonant reaction rate of charged-particle-induced reactions. The nonresonant reaction rate and the effective $S$-factor have been represented in terms of LFA. In the frame of LFA, the nonresonant reaction taken place within the Gamow window can be considered as a "resonance reaction" with a width of $\Gamma$ which is equal to that of $1 / e$ width $(\Delta)$ in a well-known Gaussian-Function Approximation (GFA).
\end{abstract}

PACS. 25.40.Lw Radiative capture

The astrophysical reaction rate of a charged-particleinduced nonresonant reaction, which is also defined as direct capture reaction, has been discussed detailedly in many works [1-3]. It is well known that the reaction rate per particle pair $\langle\sigma v\rangle$ can be written in the form of eq. (4.17) in [3], i.e.,

$\langle\sigma v\rangle=\left(\frac{8}{\pi \mu}\right)^{1 / 2} \frac{1}{(k T)^{3 / 2}} \int_{0}^{\infty} S(E) \exp \left[-\frac{E}{k T}-\frac{b}{E^{1 / 2}}\right] \mathrm{d} E$,

where all the quantities defined here follow exactly the same definitions in [3]. For a given stellar temperature $T$, nuclear reactions take place in a relatively narrow energy window around the effective burning energy of $E_{0}$ (see fig. 4.6 in [3], and the present fig. 1).

Frequently, the $S(E)$ factor is nearly a constant over the window,

$$
S(E)=S\left(E_{0}\right)=\text { const. }
$$

In this condition, eq. (1) reduces to

$\langle\sigma v\rangle=\left(\frac{8}{\pi \mu}\right)^{1 / 2} \frac{1}{(k T)^{3 / 2}} S\left(E_{0}\right) \int_{0}^{\infty} \exp \left[-\frac{E}{k T}-\frac{b}{E^{1 / 2}}\right] \mathrm{d} E$.

Although the integration can be computed by the numerical method readily, a Gaussian-Function Approximation (GFA) for the integrand in eq. (3) had been used in order to make the physics clear. The details of the approximation can be found in $[2,3]$. Briefly, the exponential term in

\footnotetext{
a e-mail: hjianjun@ph.ed.ac.uk
}

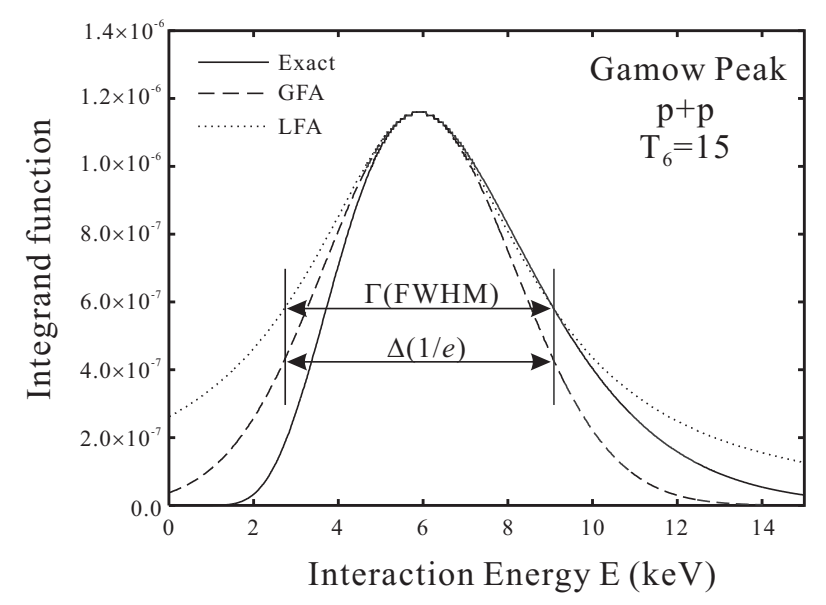

Fig. 1. Curves for the Gamow peak for the $p+p$ reaction at $T_{6}=15$, as obtained from an exact expression and from a Gaussian-Function Approximation (GFA) as well as a Lorentzian-Function Approximation (LFA). Here, $E_{0}=$ $5.89 \mathrm{keV}$ with $\Gamma=\Delta=6.37 \mathrm{keV}$.

the integrand can be fairly well approximated by a Gaussian function:

$$
\exp \left(-\frac{E}{k T}-\frac{b}{E^{1 / 2}}\right)=I_{\max } \exp \left[-\left(\frac{E-E_{0}}{\Delta / 2}\right)^{2}\right]
$$

In this approximation, the $1 / e$ width of the peak is defined as the effective width $\Delta$ of the energy window, wherein most of the reactions take place (fig. 1). This width is 
determined by matching the second derivative of eq. (4), i.e.,

$$
\Delta=\frac{4}{3^{1 / 2}}\left(E_{0} k T\right)^{1 / 2},
$$

as it is shown in fig. 1 . Actually the quantity $\Delta E_{0}$ indicated in fig. 4.6 [3] is improper, and it should be the $1 / e$ width rather than the full width at half maximum (FWHM) of the Gamow peak. And all other approximated equations, e.g., eqs. (4.26), (4.27), (4.32) and (4.34) in [3], have been deduced from this Gaussian-Function Approximation (GFA).

In the present work, a Lorentzian-Function Approximation (LFA) has been used to obtain some similar equations as those deduced from GFA. As for LFA,

$$
\exp \left(-\frac{E}{k T}-\frac{b}{E^{1 / 2}}\right)=I_{\max } \frac{(\Gamma / 2)^{2}}{\left(E-E_{0}\right)^{2}+(\Gamma / 2)^{2}} .
$$

Similarly, the full width at half maximum (FWHM) of this Lorentzian function, $\Gamma$, is determined by matching the second derivative of eq. (6), with a result of $\Gamma=\Delta$ as shown in fig. 1. In terms of LFA, the FWHM $(\Gamma)$ of the peak is defined as the effective width of the energy window, wherein most of the reactions take place. Therefore, the nonresonant reactions which have taken place within the Gamow window can be considered as a "resonance reaction" with a width of $\Gamma$, and this width, although it is equal to the $1 / e$ width of GFA (i.e., $\Delta$ ), is of a novel physical meaning.

With GFA in eq. (4), the reaction rate becomes

$$
\begin{aligned}
\langle\sigma v\rangle & \simeq\left(\frac{8}{\pi \mu}\right)^{1 / 2} \frac{1}{(k T)^{3 / 2}} S\left(E_{0}\right) \int_{-\infty}^{\infty} I_{\max } \exp \left[-\left(\frac{E-E_{0}}{\Delta / 2}\right)^{2}\right] \mathrm{d} E \\
& =\left(\frac{2}{\mu}\right)^{1 / 2} \frac{\Delta}{(k T)^{3 / 2}} S\left(E_{0}\right) \exp \left(-\frac{3 E_{0}}{k T}\right)
\end{aligned}
$$

by using a result of Gaussian integration:

$$
\int_{-\infty}^{\infty} \mathrm{e}^{-a x^{2}} \mathrm{~d} x=\sqrt{\pi / a} .
$$

It should be noted that the exact derivation of eq. (7) (i.e., eq. (4.27) in [3]) requires the lower limit of integration to be set as $-\infty$, although it is not necessarily so small, e.g., $-E_{0}$ is small enough. As discussed in the literature $[2,3]$, the exact form of $\exp \left(-\frac{E}{k T}-\frac{b}{E^{1 / 2}}\right)$ is slightly asymmetric around the energy $E_{0}$ compared with the Gaussian distribution. This general feature of the Gamow peak can be accounted for when calculating the integral in eq. (3) by the factor $F(\tau)$ :

$$
F(\tau)=1+\frac{5}{12} \frac{1}{\tau}-\frac{35}{288} \frac{1}{\tau^{2}}+\cdots,
$$

where in general the correction provided by the $1 / \tau$ is sufficient [3]. Generally speaking this correction is very small, e.g., in first order (at $\left.T_{6}=15\right)$ :

$$
\begin{aligned}
p+p: F(\tau) & =1.030, \\
{ }^{16} \mathrm{O}+{ }^{16} \mathrm{O}: F(\tau) & =1.00076 .
\end{aligned}
$$

With LFA in eq. (6), the reaction rate becomes

$$
\begin{aligned}
\langle\sigma v\rangle & \sim\left(\frac{8}{\pi \mu}\right)^{1 / 2} \frac{1}{(k T)^{3 / 2}} S\left(E_{0}\right) \int_{-\infty}^{\infty} I_{\max } \frac{(\Gamma / 2)^{2}}{\left(E-E_{0}\right)^{2}+(\Gamma / 2)^{2}} \\
& =\sqrt{\pi} \times\left(\frac{2}{\mu}\right)^{1 / 2} \frac{\Gamma}{(k T)^{3 / 2}} S\left(E_{0}\right) \exp \left(-\frac{3 E_{0}}{k T}\right) .
\end{aligned}
$$

It is obvious that the rate calculated by eq. (10) is overestimated by a factor of $\sqrt{\pi}$ compared to that calculated by GFA. Similar to GFA, a correction factor $L(\tau)$ is introduced here to account for the feature of Gamow peak, and simply

$$
L(\tau)=\frac{1}{\sqrt{\pi}} F(\tau)
$$

Therefore, using only the first-order correction the reaction rate $\langle\sigma v\rangle$, with respect to GFA and LFA, can be written in the same form (i.e., eq. (4.32) in [3])

$$
\langle\sigma v\rangle=\left(\frac{2}{\mu}\right)^{1 / 2} \frac{\Gamma(\text { or } \Delta)}{(k T)^{3 / 2}} S\left(E_{0}\right)\left(1+\frac{5}{12 \tau}\right) \exp (-\tau) .
$$

If the $S(E)$ factor is described by a slowly varying function of energy $E$ rather than by a constant, as assumed above (eq. (2)), it can be expanded in a Taylor series,

$$
S(E)=S(0)+\dot{S}(0) E+\frac{1}{2} \ddot{S}(0) E^{2}+\cdots
$$

As a result of GFA, one can replace the constant $S\left(E_{0}\right)$ factor in eq. (7) (i.e., eq. (4.27) in [3]) by an effective $S$-factor $[1,3]$ :

$$
S_{\text {eff }}\left(E_{0}\right)=S(0)\left[1+\frac{5}{12 \tau}+\frac{\dot{S}(0)}{S(0)}\left(E_{0}+\frac{35}{36} k T\right)+\frac{1}{2} \frac{\ddot{S}(0)}{S(0)}\left(E_{0}^{2}+\frac{89}{36} E_{0} k T\right)\right],
$$

while as a result of LFA, one can obtain

$$
\begin{aligned}
S_{\mathrm{eff}}^{\mathrm{LFA}}\left(E_{0}\right) & =S(0)\left\{1+\frac{\dot{S}(0)}{S(0)} E_{0}+\frac{1}{2} \frac{\ddot{S}(0)}{S(0)}\left[E_{0}^{2}+\left(\frac{\Gamma}{2}\right)^{2}\right]\right\}\left(1+\frac{5}{12 \tau}\right) \\
& =S(0)\left[1+\frac{5}{12 \tau}+\frac{\dot{S}(0)}{S(0)}\left(E_{0}+\frac{5}{36} k T\right)+\frac{1}{2} \frac{\ddot{S}(0)}{S(0)}\left(E_{0}^{2}+\frac{53}{36} E_{0} k T\right)\right] .
\end{aligned}
$$

For the general case $k T \ll E_{0}$, the difference between the present derived effective $S$-factor $\left(S_{\text {eff }}^{\text {LFA }}\left(E_{0}\right)\right)$ and the previously derived one is quite small.

In summary, a Lorentzian-Function Approximation (LFA) has been developed in calculating the nonresonant reaction rate of charged-particle-induced reactions. In the LFA frame, the nonresonant reaction which has taken place within the Gamow window can be considered as a "resonance reaction" with a width of $\Gamma$, and this width is equal to the $1 / e$ width $(\Delta)$ in a well-known GaussianFunction Approximation. This interpretation is supposed to have a novel physical meaning.

I would like to express many thanks to my wife who has been supporting me during the preparation of the manuscript.

\section{References}

1. W.A. Fowler, G.R. Caughlan, B.A. Zimmerman, Annu. Rev. Astron. Astrophys. 5, 525 (1967).

2. D.D. Clayton, Principles of Stellar Evolution and Nucleosynthesis (University of Chicago Press, Chicago, 1983).

3. C.E. Rolfs, W.S. Rodney, Cauldrons in the Cosmos (University of Chicago Press, Chicago, 1988). 